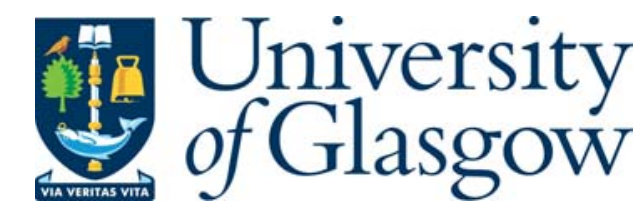

Bevan, G. and Gollee, H. and O'Reilly, J. (2007) Automatic lateral emergency collision avoidance for a passenger car. International Journal of Control 80(11):pp. 1751-1762.

http://eprints.gla.ac.uk/3455/

Deposited on: 19 October 2007 


\title{
Automatic lateral emergency collision avoidance for a passenger car
}

\author{
G. Bevan, H. Gollee and J. O’Reilly
}

This is a preprint of an article whose final and definitive form has been published in the International Journal of Control (C)2007 Taylor \& Francis; the International Journal of Control is available online at informaworld ${ }^{T M}$

\author{
Journal: International Journal of Control \\ Volume: 80 \\ Issue: $\quad 11$ \\ Date: November 2007 \\ Pages: $\quad 1751-1762$ \\ URL: http://dx.doi.org/10.1080/00207170701324271
}




\title{
Automatic lateral emergency collision avoidance for a passenger car
}

\author{
GERAINT BEVAN ${ }^{\dagger}$, HENRIK GOLLEE and JOHN O'REILLY. \\ Centre for Systems and Control, University of Glasgow, Glasgow, G12 8QQ Scotland. \\ (Received 00 Month 200x; In final form 00 Month 200x)
}

Keywords: emergency collision avoidance; steering; brakes

\begin{abstract}
Longitudinal collision avoidance controllers are of limited benefit for preventing head-on collisions between road vehicles travelling at high speed or for preventing rear end collisions when there is insufficient separation between the vehicles. In these circumstances, aggressive lateral vehicle manoeuvres are more appropriate.

This paper develops a controller architecture to perform an emergency lateral collision avoidance manoeuvre. Simulation results indicate significant improvements in collision avoidance at vehicle speeds up to $100[\mathrm{~km} / \mathrm{hr}]$ using integrated automatic steering and braking.
\end{abstract}

\section{Introduction}

As roads become busier and automotive technology improves, there is considerable potential for driver assistance systems to improve the safety of road users. It is becoming increasingly common for luxury cars to be fitted with longitudinal collision avoidance systems, where cruise control functions are integrated with forward looking obstacle detection sensors to assist deceleration of the car when necessary. Such devices are a valuable aid if an impending rear-end collision between cars travelling in the same lane is due to driver inattention and the vehicles are amply separated in space and time for the aft vehicle to brake. However, longitudinal collision avoidance systems are of limited benefit for preventing head-on collisions or avoiding obstacles which appear suddenly in front of a moving vehicle. In these circumstances, aggressive lateral manoeuvres are more appropriate; as well as altering the path of the vehicle to move it out of danger, the manoeuvre can be completed in a shorter distance than that required to stop the vehicle. Notwithstanding the availability of steer-by-wire augmentation and electronic stability programmes that could enable such manoeuvres to be initiated and performed under the guidance of a vehicle management computer, there is relatively little work reported on lateral emergency collision avoidance (Vahidi \& Eskandarian, 2004). Of particular interest, however, is the work of Shiller \& Sundar (1998) who describe how vehicles are not always able to avoid a collision solely by braking; evasive steering manoeuvres are often required, particularly at higher speeds.

There has been research into the use of steer-by-wire to perform automatic lane changing manoeuvres such as that performed under the auspices of the California PATH project but this has tended to concentrate on gentle manoeuvres undertaken by autonomous vehicles travelling on intelligent highway systems (Rajamani et al., 2000) or systems which are reliant on inter-vehicle communication (Swaroop \& Yoon, 1999). Burgio \& Zegelaar (2006) identify some of the difficulties related to integration of brake and steering controls. The problem is multi-input, multi-output (MIMO), intrinsically non-linear due to inherent tyre characteristics and suffers from a high degree of plant uncertainty due to variation in parameters. They propose a method of state feedback linearisation to produce a globally stable controller that uses the brakes only in critical cases.

\footnotetext{
†Corresponding author. Email: g.bevan@eng.gla.ac.uk
} 
Table 1. Nomenclature

\begin{tabular}{ll}
\hline \multicolumn{2}{c}{ Earth ${ }^{\oplus}$ and body axis system symbols } \\
\hline$F$ & force \\
$J$ & moment of inertia \\
$l$ & length \\
$M$ & moment about centre of gravity \\
$R$ & radius of curvature \\
$V$ & vehicle speed \\
$X$ & longitudinal position \\
$Y$ & lateral position \\
$\Psi$ & heading angle \\
$\delta$ & steering angle \\
$\phi, \theta$ & trajectory generation angles \\
\hline & Wheel axis system symbols \\
\hline$\alpha$ & lateral slip angle \\
$f$ & force \\
$v$ & wheel speed \\
\hline & Axis independent symbols \\
\hline$C_{\alpha}$ & tyre lateral stiffness \\
$g$ & gravitational acceleration \\
$m$ & vehicle mass \\
$\mu$ & road/tyre friction coefficient \\
\hline & Matrix and vector symbols \\
\hline $\mathbf{A}$ & linearised state matrix \\
$\mathbf{B}_{\delta}$ & linearised input matrix \\
$\mathbf{B}_{\mathbf{f}}$ & linearised input matrix \\
$\mathbf{f}$ & longitudinal tyre forces \\
$\mathbf{K}, \mathbf{L}$ & gain matrices \\
$\mathbf{v}$ & velocity vector \\
$\mathbf{w}$ & acceleration vector \\
$\rho$ & scheduling vector \\
$\tau$ & singular value tolerance \\
\hline & Subscripts and superscripts \\
\hline 0 & feedforward \\
$i$ & wheel index \\
$x$ & longitudinal \\
$y$ & lateral \\
\hdashline & vertical \\
$\oplus$ & Earth axis system \\
\hline &
\end{tabular}

There are several issues that remain to be addressed before it would be practical to attempt to implement a lateral collision avoidance system on a production vehicle, particularly related to sensing of the external environment and the manner in which control would pass from the driver to the vehicle computer, and then back again. This paper focusses purely on control of the vehicle dynamics to perform an emergency collision avoidance manoeuvre under the assumptions that there is a clear adjacent lane into which the vehicle may safely move and a decision to proceed has already been taken either by a vehicle management computer or a driver-initiated action. A controller is described which integrates steering and braking actions to abruptly change lanes, controlling the vehicle using large inputs while operating in highly nonlinear regions of the dynamic envelope. Specifically the paper demonstrates in a step-by-step manner:

(i) a method of generating an aggressive lane-changing trajectory while respecting acceleration limits;

(ii) the utility of feedforward control using a geometrically-derived, linear, steady-state, inverse model to cause the vehicle to follow the pre-determined trajectory;

(iii) that easily tuned hybrid proportional controllers can correct for nonlinearities, uncertainties and disturbances that would otherwise disrupt the transient and steady state performance of the feedforward controller; and

(iv) the suitability of velocity based linearisation as a technique for development of a scheduled poleplacement MIMO controller for further stabilisation about the chosen trajectory and as a means of allocating control effort between redundant actuators. 
This paper is structured as follows. Section 2 describes an obstacle avoidance manoeuvre followed by the parameters and equations of motion of a car required to perform the manoeuvre. Methods used in the development of the integrated controller are described in Section 3, including determination of an appropriate trajectory, linearisation of a non-linear vehicle model and a description of the controller architecture and control loop details. The results of evaluations performed by simulation are described in Section 4 , after which discussion and conclusions are presented.

\section{Manoeuvre and vehicle specifications}

\section{$2.1 \quad$ Emergency obstacle avoidance manoeuvre}

International standard ISO 3888 specifies test track layouts for two lateral manoeuvres for passenger cars. Part 1 specifies the track layout for a double-lane change. Part 2 specifies the layout for obstacle avoidance; this is similar to the part 1 definition but requires the manoeuvre to be conducted within more tightly constrained limits.

The standard is intended to be used to assess the handling characteristics of vehicles by drivers, but this test track layout provides a suitable manoeuvre requirement for an emergency collision avoidance controller. However, after performing an evasive manoeuvre, it is preferable that control of the vehicle should be returned to the driver rather than allowing the vehicle to return to the original lane automatically, so only the initial lane change is used to constrain the controller specification in the sequel, as shown in Figure 1. The track dimensions are given in Table 2. The standard recommends that the manoeuvre should be performed with an initial speed of $80 \pm 3[\mathrm{~km} / \mathrm{hr}]$.

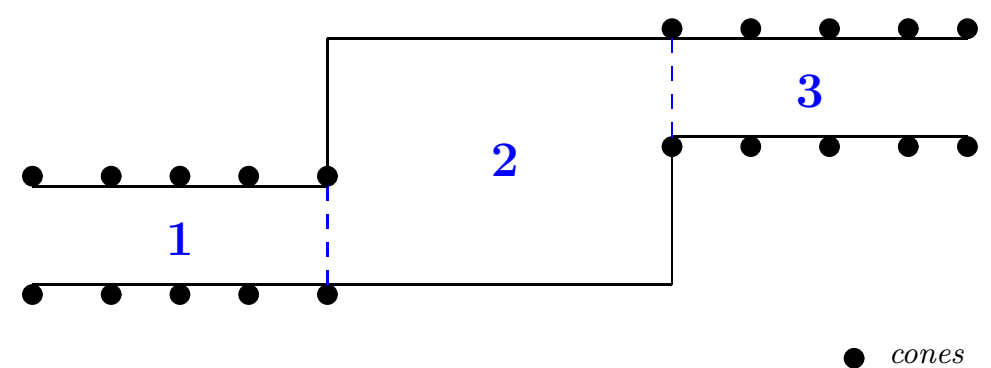

Figure 1. Test track layout for demonstration of severe lane change derived from ISO-3888-2 (2002)

Table 2. Test track dimensions based upon the layout specified in ISO-3888-2 (2002)

\begin{tabular}{ccc}
\hline Section & Length (metres) & Width (metres) \\
\hline 1 & 12.0 & $1.1 \times$ vehicle width +0.25 \\
2 & 13.5 & $2.1 \times$ vehicle width +1.25 \\
3 & 11.0 & $1.0 \times$ vehicle width +1.00 \\
\hline
\end{tabular}

\subsection{Earth, vehicle body and tyre axis systems}

Three axis systems are shown in Figure 2. The vehicle body axis system is a right-hand orthogonal axis set $(X, Y, Z)$ centred on the vehicle centre of gravity with $X$ defined positive forwards along the centre-line of the vehicle, $Y$ defined positive to the right of the vehicle and hence $Z$ defined positive downwards. An Earth-fixed axis system $\left(X^{\oplus}, Y^{\oplus}, Z^{\oplus}\right)$ is defined to be colocated and aligned with the vehicle axis at some point before the start of any manoeuvre but does not subsequently move with the vehicle. The angle of rotation between these axis systems is the vehicle heading angle, $\Psi$. For each wheel, $i$, there is a further right-hand orthogonal axis system $\left(x_{i}, y_{i}, z_{i}\right)$ centred on the wheel with $x_{i}$ defined in the direction in which the wheel is pointing. 


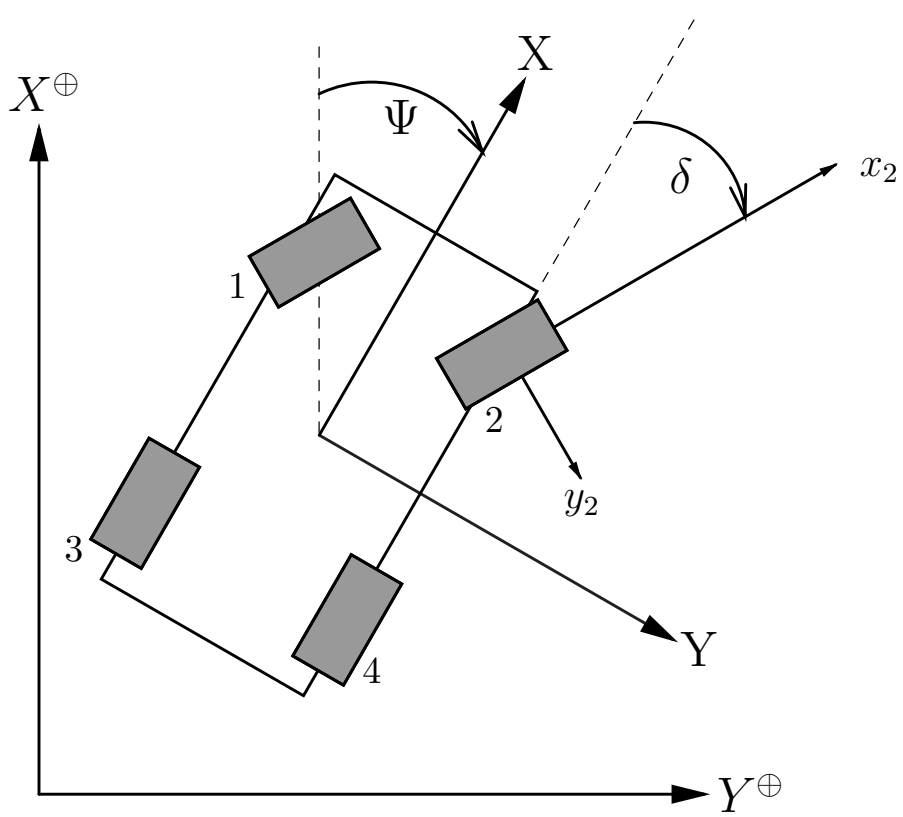

Figure 2. Earth, vehicle body and tyre axis systems

\subsection{Vehicle specification}

Development of a controller requires certain plant parameters to be specified. For this study, the plant is assumed to be a rear-wheel drive passenger car, characterised by the parameters in Table 3. Five plant

\begin{tabular}{llll} 
Table 3. & Vehicle parameters & & \\
\hline symbol & description & value & units \\
\hline$C_{\alpha}$ & lateral tyre stiffness & 10.0 & {$\left[\mathrm{rad}^{-1}\right]$} \\
$m$ & mass & 2360 & {$\left[\mathrm{~kg}^{2}\right]$} \\
$J_{z z}$ & moment of inertia & 2870 & {$\left[\mathrm{~kg} \mathrm{~m}^{2}\right]$} \\
$\left(l_{x_{1}}, l_{y_{1}}\right)$ & wheel co-ordinate, front left & $(+1.67,-0.8)$ & {$[\mathrm{m}]$} \\
$\left(l_{x_{2}}, l_{y_{2}}\right)$ & wheel co-ordinate, front right & $(+1.67,+0.8)$ & {$[\mathrm{m}]$} \\
$\left(l_{x_{3}}, l_{y_{3}}\right)$ & wheel co-ordinate, rear left & $(-1.41,-0.8)$ & {$[\mathrm{m}]$} \\
$\left(l_{x_{4}}, l_{y_{4}}\right)$ & wheel co-ordinate, rear right & $(-1.41,+0.8)$ & {$[\mathrm{m}]$} \\
\hline
\end{tabular}

inputs are available for controlling the vehicle trajectory, using the steering and braking systems. A steerby-wire system enables a front wheel steering angle $\delta$ to be obtained under electronic control; there is no rear wheel steering. An anti-lock braking system (ABS) and electronic stability programme (ESP) are installed on the vehicle, modification of which allow longitudinal braking forces to be demanded directly for each of the four wheels. In production vehicles, an ESP automatically applies differential braking forces to induce a yawing moment which acts to stabilise the vehicle yaw rate during aggressive turns. However, commercial ESPs tend to be fairly conservative and act to maintain a safety margin of the order of $10 \%$ from the theoretical limits. To take the vehicle closer to its physical limits, the existing ESP system can be disabled and its functionality commandeered to produce longitudinal forces demanded by a less conservative collision avoidance controller. In contrast, lateral forces can be demanded only indirectly by altering the steering angle of the wheels.

The vehicle is equipped with numerous sensors, including wheel speed sensors, a gyroscopic inertial navigation system (INS) and global positioning system (GPS) receiver. Accurate and frequent (100 Hz) body acceleration measurements can be obtained from the INS, which may be filtered and integrated to provide velocity and position estimates. Lower frequency $(10 \mathrm{~Hz})$ GPS measurements can be used to correct for sensor drift in the INS. An on-board observer can collate the various sensor data and provide timely best estimates of the vehicle states.

The most significant limit on the controlled inputs is saturation of the traction provided by the tyres, which acts as a limit on the combined force capable of being exerted by the steering and braking systems. 
In the absence of aerodynamic control surfaces or propulsive devices, the only controllable forces that may be used to accelerate a vehicle are the frictional forces generated in the small contact areas where tyre rubber meets the road surface. The frictional force that can be generated is constrained to be less than or equal to the product of the coefficient of friction $\mu$ and the normal load acting at the point of contact.

During an aggressive lateral manoeuvre there will be significant load transfer with the inner wheels becoming more lightly loaded, and thus able to produce less traction. However, this will be compensated by the outer wheels becoming more heavily loaded, and thus able to produce greater traction. The total tractive force that may be produced by the vehicle is not therefore significantly altered by this behaviour unless there is considerable variation in the condition of the road beneath each wheel. However, the maximum tractive force is directly proportional to the coefficient of friction $\mu$ between the rubber of the tyres and the road surface. Consequently $\mu$ is a fundamental parameter affecting vehicle handling.

The value of $\mu$ can vary considerably depending on the road surface material and prevailing conditions, ranging from less than 0.05 for ice to approximately 1.0 for dry asphalt (Germann et al., 1994). The total vehicle load acting over the total contact area must be equal to the vehicle weight and so the maximum acceleration that can be achieved is $\mu g\left[\mathrm{~m} / \mathrm{s}^{2}\right]$ where $g$ is the acceleration due to gravitation. In practice, the highly nonlinear characteristics of tyres mean that this level of acceleration can only be achieved longitudinally with a precise (tyre-specific) amount of slip between the tyre and the road; regulating wheel slip to achieve peak acceleration is the basis of anti-lock braking systems (ABS) and traction control systems (TCS).

The maximum achievable lateral force for a wheel is usually slightly less than the maximum longitudinal force, with the limiting value in any direction being described by a tyre-dependent friction ellipse. However, given the high uncertainty in $\mu$ and the ability for the front wheels to be steered, a reasonable approximation for the tractive force available for the vehicle as a whole, rather than for any particular tyre, is a friction circle. The most important result of the limit on the forces generated between the tyre and road is that any braking forces reduce the traction available for steering and vice versa. Thus in an evasive manoeuvre, there is a trade off between braking - which is the only way of removing energy from the system - and steering to avoid an obstacle.

There are also limits inherent in each of the systems independently, namely communication delays, sample rate limits and actuator rate limits. Additionally, the brake system is only able to satisfy a demanded longitudinal force if the wheel is not locked, but lock detection is not instantaneous. These limits imposed by sensors and actuators are specified in tables 4 and 5 .

Table 4. Steering limits

\begin{tabular}{lll}
\hline description & value & units \\
\hline communication delay & 40 & {$[\mathrm{~ms}]$} \\
sample rate & 100 & {$[\mathrm{~Hz}]$} \\
rate limit & \pm 160 & {$[\mathrm{rad} / \mathrm{s}]$} \\
\hline
\end{tabular}

Table 5. Braking limits

\begin{tabular}{lll}
\hline description & value & units \\
\hline communication delay & 20 & {$[\mathrm{~ms}]$} \\
sample rate & 50 & {$[\mathrm{~Hz}]$} \\
rate limit & +500 & {$[\mathrm{bar} / \mathrm{s}]$} \\
& -2000 & {$[\mathrm{bar} / \mathrm{s}]$} \\
wheel lock detection & $\leq 40$ & {$[\mathrm{~ms}]$} \\
\hline
\end{tabular}

\subsection{Equations of motion}

For each wheel, $i=\{1,2,3,4\}$, the longitudinal and lateral forces in the wheel axis system can be resolved into contributions to the longitudinal and lateral forces acting on the vehicle in the vehicle body axis 
system $F_{x_{i}}$ and $F_{y_{i}}$

$$
\left(\begin{array}{l}
F_{x_{i}} \\
F_{y_{i}}
\end{array}\right)=\left(\begin{array}{l}
+\cos \delta_{i},-\sin \delta_{i} \\
-\sin \delta_{i},+\sin \delta_{i}
\end{array}\right)\left(\begin{array}{l}
f_{x_{i}} \\
f_{y_{i}}
\end{array}\right)
$$

where $f_{x_{i}}$ and $f_{y_{i}}$ are the longitudinal and lateral forces in the tyre axis system and $\delta_{i}$ is the wheel steering angle. These forces can in turn be transformed into the component forces in the Earth axis system $F_{x_{i}}^{\oplus}$ and $F_{y_{i}}^{\oplus}$ and contributions to the moment about the vehicle centre of gravity $M_{z_{i}}^{\oplus}$

$$
\left(\begin{array}{c}
F_{x_{i}}^{\oplus} \\
F_{y_{i}}^{\oplus} \\
M_{z_{i}}^{\oplus}
\end{array}\right)=\left(\begin{array}{c}
+\cos \Psi,-\sin \Psi \\
-\sin \Psi,+\sin \Psi \\
-l_{y_{i}}, \quad+l_{x_{i}}
\end{array}\right)\left(\begin{array}{c}
F_{x_{i}} \\
F_{y_{i}}
\end{array}\right)
$$

where $l_{x_{i}}$ and $l_{y_{i}}$ are the co-ordinates of the wheel relative to the vehicle centre of gravity.

The vehicle has no rear wheel steering and the steer-by-wire system permits only a single steering angle $\delta$ to be set for both front wheels. Labelling the wheels: 1) front left; 2) front right; 3) rear left; 4) rear right, the steering angles for each wheel are $\delta_{1}=\delta_{2}=\delta$ for the front wheels, and $\delta_{3}=\delta_{4}=0$ for the rear.

Tyre forces are a complex function of the tyre material properties and the degree of slip between the tyre and the road surface. Many tyre models have been published, e.g. Pacejka \& Bakker (1993); Canudas de Wit \& Tsiotras (1999). However, many modern cars include an anti-lock braking system and/or electronic stability programme which regulates the tyre forces produced and can in principle be used to obtain a desired longitudinal force at each wheel to satisfy higher level control requirements.

There are no equivalent systems to regulate the lateral forces produced by the tyres.

The tyre forces are likely to saturate during an aggressive manoeuvre. However, for the purposes of controller design it is not necessary to use a highly detailed model of the tyre dynamics. Furthermore, given the wide variation in tyre characteristics and uncertainty in tyre condition at any time, it would be inadvisable for a vehicle dynamics controller to rely too heavily on detailed models of tyre behaviour. Thus, as long as the total tractive limit is adhered to, it is reasonable to assume that the lateral force $f_{y_{i}}$ generated by any tyre is proportional to its lateral slip $\alpha_{i}$, which is defined as

$$
\alpha_{i}=\arctan \frac{v_{y_{i}}}{v_{x_{i}}}
$$

where $v_{x_{i}}$ and $v_{y_{i}}$ are the longitudinal and lateral speeds of the wheel (in its own axis system), which can be defined in terms of the vehicle body velocities.

$$
\begin{aligned}
& v_{x_{i}}=\left(\dot{X}-l_{y_{i}} \dot{\Psi}\right) \cos \delta_{i}+\left(\dot{Y}+l_{x_{i}} \dot{\Psi}\right) \sin \delta_{i} \\
& v_{y_{i}}=\left(\dot{X}-l_{y_{i}} \dot{\Psi}\right) \sin \delta_{i}+\left(\dot{Y}+l_{x_{i}} \dot{\Psi}\right) \cos \delta_{i}
\end{aligned}
$$

The lateral force can then be approximated as

$$
f_{y_{i}} \approx \mu C_{\alpha} \alpha_{i} f_{z_{i}}
$$

where $f_{z_{i}}$ is the vertical load on the wheel and $C_{\alpha}$ is a tyre-specific parameter known as the lateral stiffness. 
Applying Newton's second law then gives the vehicle body longitudinal, lateral and yaw accelerations as

$$
\begin{aligned}
\ddot{X}^{\oplus} & =\frac{1}{m} \sum_{i=1}^{4} F_{x_{i}}^{\oplus} \\
\ddot{Y}^{\oplus} & =\frac{1}{m} \sum_{i=1}^{4} F_{y_{i}}^{\oplus} \\
\ddot{\Psi} & =\frac{1}{J_{z z}} \sum_{i=1}^{4} M_{z_{i}}
\end{aligned}
$$

where $m$ is the vehicle mass and $J_{z z}$ the moment of inertia about its vertical axis.

\section{Methods}

\subsection{Trajectory generation}

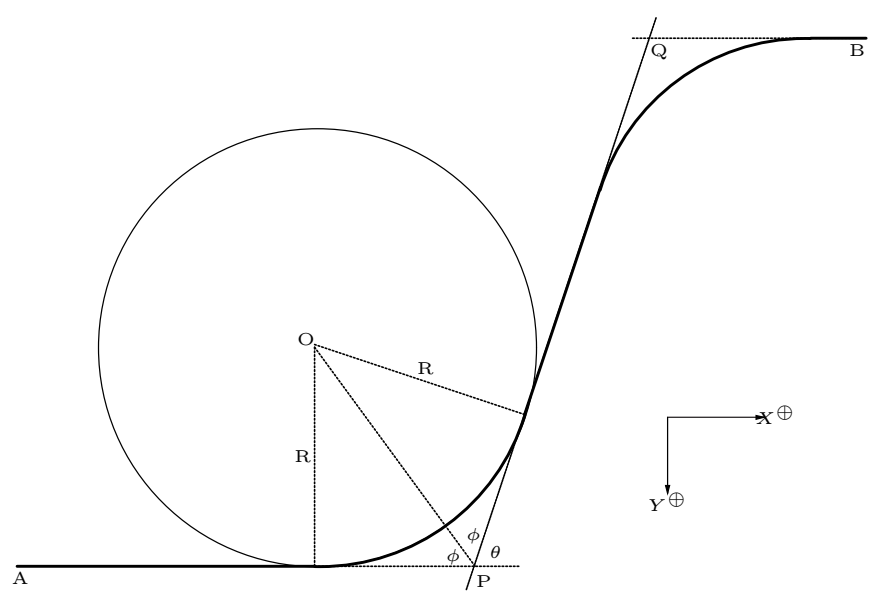

Figure 3. Trajectory generation

Given the traction limits described in Section 2.3 it is necessary to determine a trajectory that will respect the limits on the maximum acceleration of which the car is capable. Following the discussion in Section 1 of the benefits of steering over braking when travelling at high speed, it is clear that the manouevre should rely on steering as the primary control effort to navigate the vehicle through the manoeuvre. The brakes can then be used to refine the control, enabling peak performance to be extracted and corrections to be applied. This corresponds with intuition; redirecting the considerable forward momentum by pointing the car in a different direction will allow a lateral shift to be performed far faster than attempting to reduce this momentum while increasing lateral momentum.

A good reference trajectory for emergency collision avoidance will therefore aim to turn the car as quickly as possible at the start of the manoeuvre, use the vehicle's forward speed to move swiftly into the adjacent lane before aggressively redirecting its momentum in the direction of the new lane. It should be noted that this is significantly different from the more gentle manoeuvres investigated for lane-changing manouevres by vehicles on autonomous highway systems where passenger comfort is of greater importance.

Determination of an optimal trajectory generally requires a large amount of calculation (Hattori et al., 2006). However, it is possible to derive a good trajectory by making some simplifying assumptions and considering the physical limits of the vehicle. The vehicle is capable of a maximum acceleration of $\mu g\left[\mathrm{~m} / \mathrm{s}^{2}\right]$ and if steering is to be preferred over braking, it is sensible to direct the acceleration vector 
perpendicular to the forward speed of the car. This will result in a circular trajectory, with radius of curvature $R=V^{2} /(\mu g)[\mathrm{m}]$ where $V$ is the vehicle speed.

Figure 3 shows how such a trajectory may be calculated to move from point $A$ to $B$. Specification of the points $P$ and $Q$ allows the co-ordinates at which the vehicle must begin and end turning to be calculated. If $\theta$ is the arctangent of the gradient of the line joining $P$ and $Q$, the car must begin turning at distance $X^{\oplus}=X_{P}^{\oplus}-\frac{R}{\tan \phi}$ and stop turning at $X^{\oplus}=X_{P}^{\oplus}+\frac{R \cos \theta}{\tan \phi}$ before turning again at $X^{\oplus}=X_{Q}^{\oplus}-\frac{R \cos \theta}{\tan \phi}$ and straightening its heading at $X^{\oplus}=X_{Q}^{\oplus}+\frac{R}{\tan \phi}$ where $\phi=\frac{\pi}{2}-\frac{\theta}{2}$.

Given this curve definition and by choosing to keep the car pointing tangentially to the curve throughout the manoeuvre, $\Psi_{\text {ref }}=\arctan \left(\frac{\mathrm{d} Y_{\text {ref }}^{\oplus}}{\mathrm{d} X^{\oplus}}\right)$, reference lateral and yaw velocities for the vehicle can be defined as functions of longitudinal position $X^{\oplus}$ (in the Earth axis system) and the vehicle body velocities (longitudinal $\dot{X}$, lateral $\dot{Y}$ and yaw $\dot{\Psi}$ ) by rotating the co-ordinate axes.

$$
\begin{aligned}
& \dot{X}^{\oplus}=\dot{X} \cos \Psi-\dot{Y} \sin \Psi \\
& \left(\begin{array}{c}
\dot{Y}_{r e f}^{\oplus} \\
\dot{\Psi}_{r e f}^{\oplus}
\end{array}\right)=\left(\begin{array}{c}
\frac{\mathrm{d} Y_{r e f}^{\oplus}}{\mathrm{d} X^{\oplus}}\left(X^{\oplus}\right) \\
\frac{\mathrm{d} \Psi_{r e f}^{\oplus}}{\mathrm{d} X^{\oplus}}\left(X^{\oplus}\right)
\end{array}\right) \dot{X}^{\oplus} \\
& \left(\begin{array}{c}
\dot{X}_{r e f} \\
\dot{Y}_{r e f} \\
\dot{\Psi}_{r e f}
\end{array}\right)=\left(\begin{array}{ccc}
\cos \Psi, & \sin \Psi, 0 \\
-\sin \Psi, & \cos \Psi, 0 \\
0 \quad, & 0,1
\end{array}\right)\left(\begin{array}{c}
\dot{X}^{\oplus} \\
\dot{Y}_{r e f}^{\oplus} \\
\dot{\Psi}_{r e f}^{\oplus}
\end{array}\right)
\end{aligned}
$$

\subsection{Vehicle model: velocity based linearisation}

The vehicle model developed in Section 2.4, equations (1) to (4), describes the vehicle acceleration as a function of its velocity, front wheel steering angle and longitudinal tyre forces

$$
\dot{\mathbf{v}}=\mathbf{g}(\mathbf{v}, \delta, \mathbf{f})
$$

Vector $\mathbf{v}$ comprises three body velocities: $\dot{X}, \dot{Y}$ and $\dot{\Psi}$, each defined in the vehicle axis system; vector $\mathbf{f}$ comprises the longitudinal tyre forces generated by each wheel, defined in the axis systems of the wheels.

Despite the simplifying assumption that the lateral tyre force is proportional to lateral slip, the model remains highly non-linear. In addition to the trigonometric nonlinearities introduced by the front wheel steering angle $\delta$ and the vehicle heading angle $\Psi$, there is a severe complication resulting from the multiplication of inputs to the front wheels: $f_{x_{i}} \times \cos \delta$ and $f_{x_{i}} \times \sin \delta$ from Equation (1).

Derivation of a linear model provides access to a wide range of linear control theoretic techniques for analysis and design. Such models are often derived by assuming small perturbations about equilibrium conditions but this would not generate an appropriate design model in this case. Lateral emergency collision avoidance requires large, abrupt steering inputs and the vehicle will operate far from equilibrium conditions for most of the manoeuvre.

Velocity-based linearisation (Leith \& Leithead, 1998) provides a method of generating linear models that are applicable away from equilibrium conditions and has been shown to be appropriate for modelling high performance vehicles (Leith et al., 2001). Partial differentiation of the nonlinear vehicle model at any operating condition with respect to time yields a linear model

$$
\ddot{\mathbf{v}} \approx \frac{\partial \mathbf{g}}{\partial \mathbf{v}} \dot{\mathbf{v}}+\frac{\partial \mathbf{g}}{\partial \delta} \dot{\delta}+\frac{\partial \mathbf{g}}{\partial \mathbf{f}} \dot{\mathbf{f}}
$$

A scheduled family of models can therefore be defined in terms of an acceleration vector $\mathbf{w}=\dot{\mathbf{v}}(\rho)$ by

$$
\dot{\mathbf{w}}=\mathbf{A}(\rho) \mathbf{w}+\mathbf{B}_{\delta}(\rho) \dot{\delta}+\mathbf{B}_{\mathrm{f}}(\rho) \dot{\mathbf{f}}
$$


where $\mathbf{A}(\rho)=\frac{\partial \mathbf{g}}{\partial \mathbf{v}}(\rho), \mathbf{B}_{\delta}(\rho)=\frac{\partial \mathbf{g}}{\partial \delta}(\rho), \mathbf{B}_{\mathrm{f}}(\rho)=\frac{\partial \mathbf{g}}{\partial \mathbf{f}}(\rho)$ and the scheduling vector $\rho$ comprises the vehicle velocity vector $\mathbf{v}$ and the front wheel steering angle $\delta$.

\subsection{Controller architecture}

Initially a collision avoidance manoeuvre is specified. In this case the manoeuvre is that described in Section 2 but this could be replaced by one derived from a vehicle's sensing of its external environment to identify gaps in the traffic. The manoeuvre specification is then passed to a trajectory generator which calculates the position, velocity and acceleration profiles for the vehicle to execute. The profiles are scheduled as a function of longitudinal position, as described in Section 3.1.

The basic collision avoidance control strategy is to use feedforward control to force the vehicle to follow a pre-determined trajectory. Feedback control is then used to stabilise the vehicle about the reference trajectory and to correct for errors that accumulate for the usual reasons: disturbances, noise, parametric uncertainties and unmodelled dynamics.

There are two distinct phases to the complete collision avoidance manoeuvre that is to be performed by the vehicle. These can be characterised as lane changing and lane keeping. The control architecture for

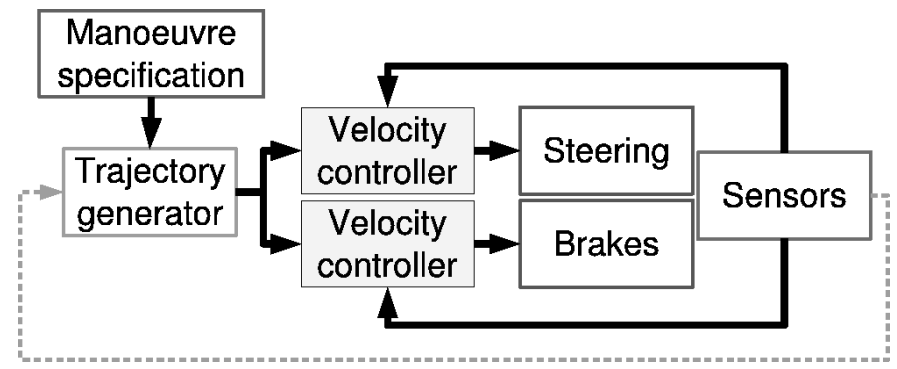

(a) lane changing

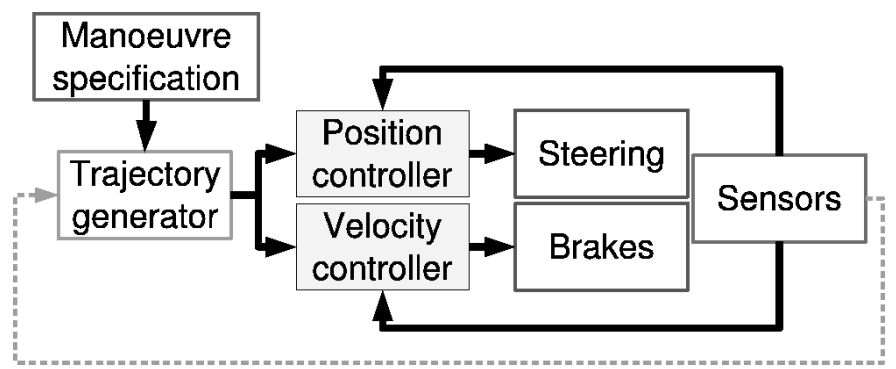

(b) lane keeping

Figure 4. Controller architecture

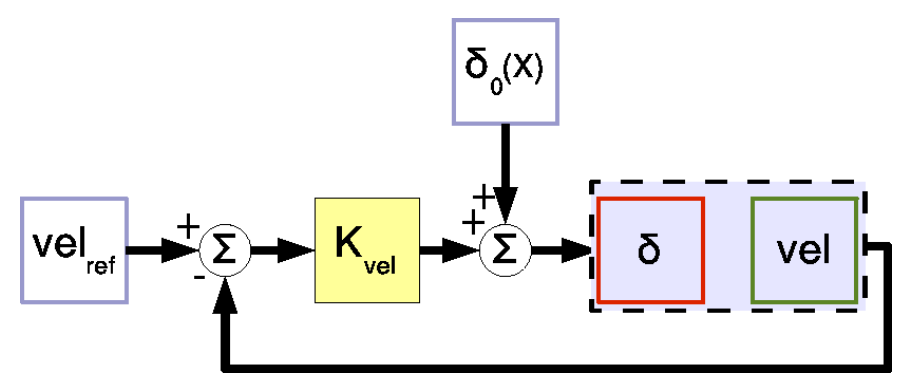

(a) lane changing

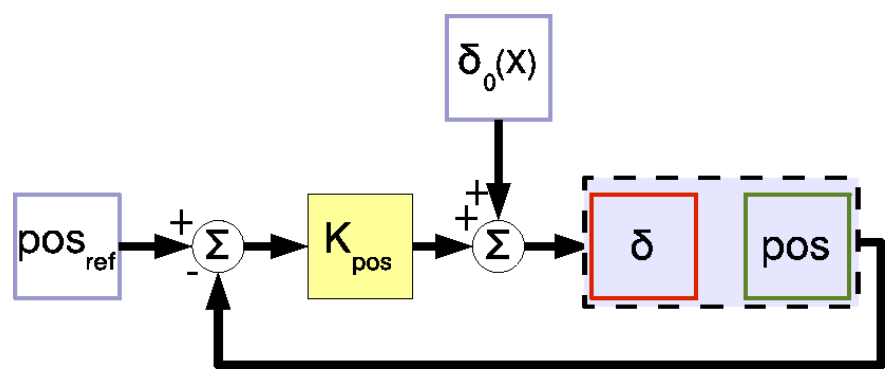

(b) lane keeping

Figure 5. Steering control loop

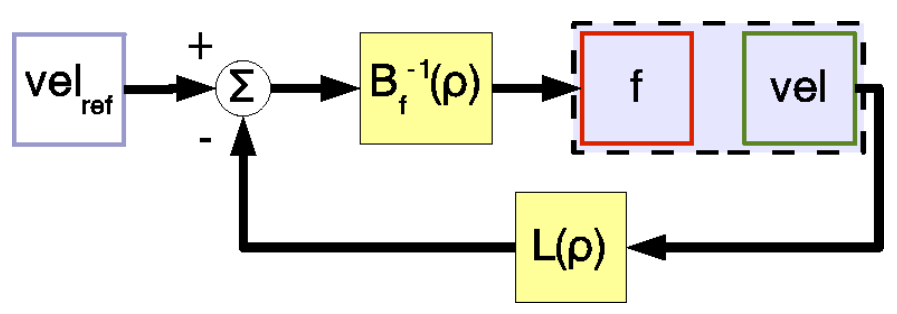

Figure 6. Brake control loop

each phase of the manoeuvre is as shown in Figure 4. Two parallel loops are used to control the vehicle dynamics: a steering loop, shown in Figure 5; and a braking loop, shown in Figure 6. 
During the lane changing phase the vehicle is turning aggressively and close control of the vehicle dynamics is required. During this first phase of the manoeuvre, all feedback control is based on the vehicle velocity to ensure that the vehicle dynamics vary as little as possible from that required to correctly follow the specified trajectory. There is little scope for correction of any accumulated errors in vehicle position or heading because the vehicle is already accelerating close to its physical limits. Subsequently, only gentle manoeuvering is necessary to correct for any errors in the final position of the vehicle and to ensure that it is on an appropriate heading to remain safely in the final lane. During this second phase of the collision avoidance manoeuvre, the vehicle position and heading angle are therefore fed back to the steering controller to allow the appropriate corrections to be made. Throughout the entire collision avoidance manoeuvre, the brake control loop uses feedback to stabilise the vehicle velocity about the reference trajectory, acting primarily through tight control of the vehicle yaw rate.

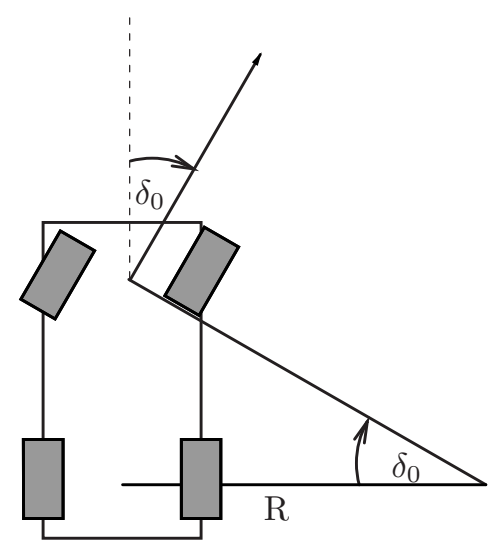

Figure 7. Ackermann steering angle $\delta_{0}$ is used as a feedforward control input

The main component of the steering control loop is feedforward of a nominal steering angle $\delta_{0}$. This steering angle is derived from the Ackermann steering angle (Milliken \& Milliken, 1995) (also known as the wheelbase angle) for a bicycle model; it is the geometrically-determined angle that would cause the vehicle to follow the specified trajectory at low speed and in the absence of wheel slip if the wheels were located along the vehicle centre-line. Figure 7 shows how this steering angle may be calculated for a vehicle which pivots about its rear wheels with no slip, using the vehicle wheelbase and the radius of curvature during the turn.

$$
\delta_{0}=\arctan \frac{l_{x_{1}}-l_{x_{3}}}{R}
$$

The feedforward component of the steering angle is augmented by a small feedback element, proportional to either velocity error or position error depending on the phase of the manoeuvre. Simple proportional control is adequate for the task and has the considerable benefit that it may easily be tuned by engineers in response to test data after the feedforward steering profile and brake control loop have been designed. As noted previously, there is a high degree of interaction between the steering and braking of the vehicle and the system is not therefore amenable to traditional linear analysis techniques for choosing the gain. However, as it is only necessary to select a single parameter in each of the two phases of the manoeuvre, it is feasible to perform a simple line-search of the parameter space with vehicle simulations in a short time once the other elements of the controller have been implemented.

The primary purpose of the brake control loop is to provide yaw rate correction to stabilise the vehicle velocity about its reference trajectory. The design of this controller is complicated by virtue of being for a multi-input, multi-output (MIMO) system with redundant actuators, for which it is not possible to assign any actuator to have primary responsibility for any of the outputs that are to be controlled.

To solve the problem of allocation of control effort between the four brakes, the controller includes a control allocation block $\mathbf{B}_{\mathrm{f}}^{-1}(\rho)$ which weights the control inputs according to the relative effect that each actuator has on the velocity error signals that are being controlled. It is obtained by calculating 
the pseudo-inverse of $\mathbf{B}_{\mathrm{f}}(\rho)$, that part of the velocity-based linearisation (Equation (5)) which relates the longitudinal forces to the model rates. It is necessary to use a pseudo-inversion rather than a true matrix inversion because the matrix is non-square; three control signals are allocated to four actuators.

When performing a pseudo-inversion of a matrix, a tolerance $\tau$ must be specified. Singular values less than $\tau$ are equated to zero during the pseudo-inversion procedure and choosing an appropriate value can have a significant impact on the suitability of the final inversion; in particular, affecting the resultant presence or absence of numerical difficulties that may arise due to calculations involving very small or large numbers. Observing that the matrix norm of a pseudo-inverse of a matrix is equal to the reciprocal of its smallest eigenvalue, $\tau$ can thus be used to place useful bounds on the norm of the pseudo-inversion.

$$
\left\|\mathbf{B}_{\mathrm{f}}^{-1}(\rho)\right\|=\frac{1}{\lambda} \quad \text { where } \lambda=\min \operatorname{eig}\left(\mathbf{B}_{\mathrm{f}}(\rho)\right): \lambda \geq \tau
$$

and hence

$$
\left\|\mathbf{B}_{\mathrm{f}}^{-1}(\rho)\right\| \leq \frac{1}{\tau}
$$

Good results have been obtained using a tolerance $\tau=4 /(m g)$, which is approximately equal to the reciprocal of the greatest force that each tyre can produce on dry asphalt if the weight of the car is evenly distributed. It should be noted that this tolerance is many orders of magnitude greater than the default value used in matrix algebra tools such as Matlab and Octave, in which values comparable to the machine precision are typical. The result of this bound on the force allocation matrix is that a control effort with unity-magnitude will result in forces close to the system limits, while greater values are likely to lead to saturation of the tractive force. Physical insight into the system therefore makes it a simple matter to place poles or select suitable gains for this loop.

The output of the control allocation block may be positive or negative. Combination of engine torque with independent braking could potentially allow a vehicle to produce positive or negative wheel forces on demand. However, the brakes on this vehicle are only capable of producing retarding torques so positive outputs from the block are set to zero.

The brake control loop also contains a scheduled gain matrix $\mathbf{L}(\rho)$, which is designed to give the vehicle uniform dynamic behaviour as its operating condition changes. After obtaining the state matrix of the velocity-based linearisation at any given condition, standard pole placement is used to calculate the matrix elements. The presence of $\mathbf{B}_{\mathrm{f}}^{-1}(\rho)$ before the plant means that the identity matrix can be used instead of the real input matrix during the pole placement procedure. The poles are placed by identifying the natural open loop poles of the car when driven straight ahead at low speed and causing them to remain at these locations no matter how the vehicle speed and steering angle change. Natural limits exist on the value of the values of the resulting gains. If too low, the controller will obviously be ineffective and provide insufficient control of yaw rate. If the gain is too high, the tyre forces will saturate, thus reducing the effectiveness of the controller for inducing a yawing moment. A well-tuned controller for operating the vehicle at its physical limit should therefore produce forces just below the saturation threshold of the tyres during the specified manoeuvre.

It is not necessary to explicitly control the longitudinal velocity of the vehicle and attempting to do so would reduce the available traction that can be used to provide lateral acceleration. Therefore, the error signal for this output is set to zero by equating the reference velocity $\dot{X}_{r e f}$ to the measured value $\dot{X}$. Thus the braking force is used only to control the yaw and lateral velocities of the vehicle. Nevertheless, the vehicle will decelerate slightly as a consequence of brake operation. Consequently, the minimum radius of turn that the vehicle is capable of achieving will reduce as the manoeuvre progresses and lower lateral forces will be required of the tyres to follow the desired trajectory. Thus, the trajectory remains feasible despite the possibility of some tyre saturation resulting from combined steering and braking. 


\section{Results}

The non-linear vehicle model developed in Section 2.4 (equations (2) to (4)) was implemented in C++ with interfaces to Octave and Matlab to provide a means of evaluating the controller by simulation. In addition to the vehicle body dynamics, this evaluation model includes first-order lags and pure delays to represent the dynamics and communications delay inherent in the actuators. The model also includes random perturbations of up to $10 \%$ which may be applied to the system states, inputs, outputs and linearised state matrices to simulate the disturbances, noise and uncertainties described in Section 3.3.

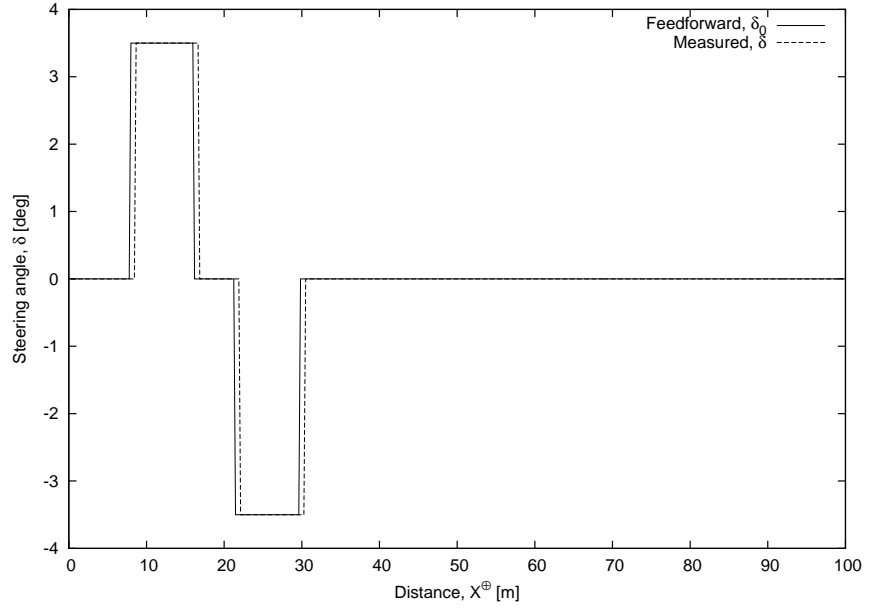

(a) Steering angle

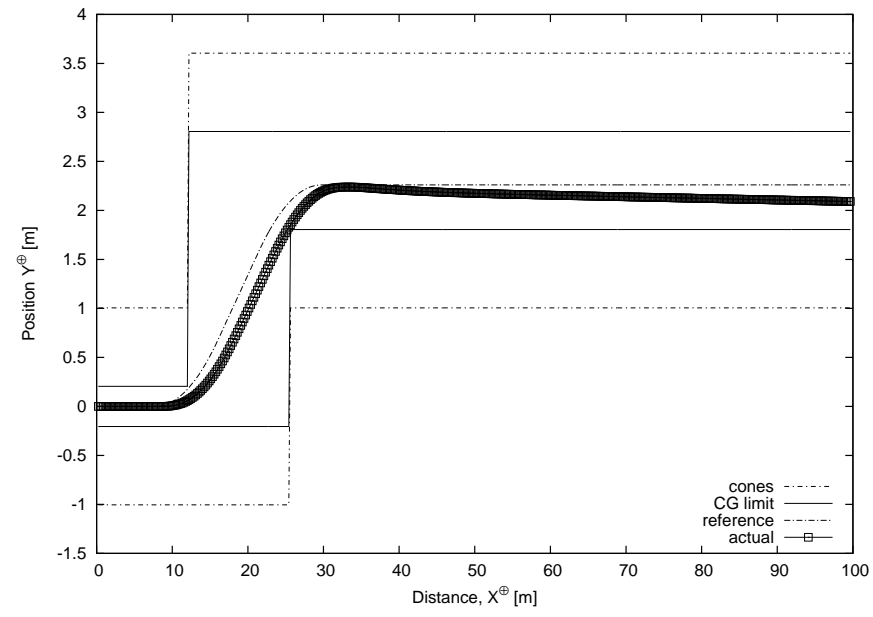

(b) Vehicle trajectory

Figure 8. Avoidance manoeuvre simulation at $80 \mathrm{kph}$ using feedforward steering

Simulations were performed over a range of speeds from $40[\mathrm{~km} / \mathrm{hr}]$ to $100[\mathrm{~km} / \mathrm{hr}]$. Results for simulations performed with an initial velocity of $80[\mathrm{~km} / \mathrm{hr}]$, the speed specified for performance of the manoeuvre in Section 2, and with a uniform coefficient of friction $\mu=1$ are shown in Figure 8 for the feedforward controller acting alone and in Figure 9 for the integrated feedforward and feedback controllers acting in concert.

Figures 8(a) and 9(a) show the feedforward steering angle $\delta_{0}$ and actual output angle $\delta$ in each case. The feedforward profile is clearly seen as two sharp pulses corresponding to the two turns in the reference trajectory. Figures 8(b) and 9(e) show the trajectory followed by the vehicle. The dashed outer lines indicate the positions of the cones described in the manoeuvre specification; the parallel solid inner lines show the approximate limits within which the vehicle centre of gravity must remain if the vehicle is to avoid hitting the cones, although this is dependent on the vehicle orientation.

Figure 9(b) shows the longitudinal forces demanded by the brake controller during the simulation in which it is active, while figures $9(\mathrm{c})$ and $9(\mathrm{~d})$ show the corresponding output velocities and accelerations of the vehicle centre of gravity: longitudinal, lateral and yaw.

\section{Discussion}

It can be seen in Figure 8(a) that the output steering angle $\delta$ slightly trails the reference value $\delta_{0}$ because of communication delay and actuator lag. The corresponding trajectory plot (Figure 8(b)) shows that this feedforward control provides a reasonable means of causing the vehicle to change lane, but its performance is not perfect. Small errors due to the discrete nature of the digital controller and disturbances to the states and inputs result in the vehicle not accurately tracking the centre-line of the newly acquired lane following the lane change. Simple proportional control of the vehicle heading angle and lateral position is adequate to correct for this, hence the implementation of the proportional lane keeping controller (Figure 5(b)) which augments the feedforward steering angle based on the sum of the errors in these signals. More challenging to address is the lag within the system due to communication delay and actuator dynamics. The delay 
results in the vehicle only just avoiding excession of the specified collision avoidance manoeuvre limits at $80[\mathrm{~km} / \mathrm{hr}]$. Compensation for this lag is the reason that the velocity controllers are required to operate during the lane change phase of the manoeuvre.

The action of the brake controller is shown in Figure 9(b). The first feature to note is that all of the brake forces produced are well within the range that might feasibly be produced by the tyres (approximately 0 to $-6000[\mathrm{~N}])$. This is a result of the specification of the tolerance during the pseudo-inversion procedure. Reducing the tolerance has the effect of increasing the forces demanded by the controller. Qualitatively the controller performs exactly as desired. The manoeuvre requires a turn to the right followed by a turn to the left (remembering that $Y$ and $Y^{\oplus}$ are defined to be positive to the right). During the initial turn to the right, the brakes on the right of the vehicle are active and therefore assist the manoeuvre by causing the vehicle to yaw in the direction of the turn, in the same way that vehicles with caterpillar tracks turn by reducing the speed of the inside track. Similarly during the subsequent turn to the left, it is the brakes on the left side of the vehicle which are active, again assisting the turn by causing the vehicle to yaw in the appropriate direction.

Consideration of the contributions of individual braking forces to the overall vehicle yawing moment reveals that the front/rear behaviour of the brakes is also as desired. During a manoeuvre, the front wheels point into the direction of the turn and consequently a small component of any braking force on these wheels acts in the opposite direction, resisting the turn. The pseudo-inverse matrix in the brake control loop has correctly accounted for this by slightly increasing the rear wheel braking forces at the expense of the front wheel braking forces.

The braking profile is echoed by the proportional component of the steering control loop during the lane change manoeuvre, which is expected because both control loops are acting on identical error signals. Interaction between the control effort of each loop is accounted for by the presence of $\delta$ in the scheduling vector $\rho$ which is used within the generation of the control allocation matrix. As the steering angle is increased, a greater proportion of the braking effort transfers to the rear wheels. In comparison to a design comprising two entirely independent controllers, this reduction of longitudinal braking forces as the steering angle - and hence front lateral tyre force - increases provides considerable benefits, namely:

(i) a reduction in the risk of exceeding the total traction limit available to the wheel;

(ii) a reduction in the risk of the brake controller compromising the effectiveness of the steering controller by excessive reduction of the front wheel speed; and

(iii) a reduction in the risk of the steering controller compromising the effectiveness of the brake controller by directing the applied brake force in the wrong direction.

These benefits arise automatically from the use of a scheduled, inverse model within the controller. It would be desirable to design them into any integrated steering and braking control system.

The combined effect of the integrated controllers is demonstrated in the vehicle trajectory plot of Figure 9 (e) where it can be seen that there is a far greater longitudinal margin than in the equivalent trajectory shown in Figure 8(b). The net result of the improvement in performance is that the vehicle is capable of successfully performing the emergency collision avoidance manoeuvre when the speed is increased up to $100[\mathrm{~km} / \mathrm{hr}]$, something that is not possible if the velocity controllers are removed from the system.

A significant benefit of the use of simple geometric models for determination of the reference vehicle trajectory and feedforward steering angle arises from the insensitivity of the method to plant uncertainty. In particular, it is not necessary to have an accurate measurement of the vehicle mass or mass distribution, quantities which can vary considerably depending on the loading of the vehicle. Nor do the methods rely on having a highly accurate model of the tyre force characteristics, data which are very expensive and timeconsuming to obtain. Trajectory generation is dependent only on an estimate of $\mu$, the friction coefficient; the feedforward steering profile relies solely on the longitudinal position of the front wheels relative to the centre of gravity, a value which is known within a reasonable margin to a high degree of certainty.

Figure 9(d) shows that the vehicle acceleration remains well within the limit of $\mu g=9.81[\mathrm{~m} / \mathrm{s}]$ specified in the trajectory generation routine. Thus it may be inferred that the linear model used to develop the steering angle model is somewhat conservative. More aggressive performance may be achievable by accounting for some of the more significant nonlinearities in the steering system when generating the 
feedforward profile.

\section{Conclusions}

This paper reports on the development of a controller to perform an emergency lateral collision avoidance manoeuvre. The simulation results indicate significant improvements in collision avoidance at vehicle speeds up to $100[\mathrm{~km} / \mathrm{hr}]$ using integrated automatic steering and braking.

The overall controller architecture is structured such that each step of the design process simplifies subsequent control tasks. The trajectory generator determines a feasible reference profile from which a feedforward steering profile can be determined early in the design process without highly detailed knowledge of the vehicle dynamics. This feedforward controller performs the bulk of the servo-control effort and provides adequate control on its own. A more complex design method is required to develop the brake control loop but as this is required only for stabilisation of the vehicle velocity about its profile, model uncertainties do not have an unduly adverse effect on the overall controller performance. Finally, the feedback steering control loop reduces the effort required of the brake controller, while its simple gain matrix provides an easy way for the overall controller performance to be fine-tuned in response to experimental test data.

Further work is necessary before it would be practical to implement this controller on a production vehicle. In particular, investigation is required into the decision making process for manoeuvre initiation. Other factors worthy of consideration are how control of the vehicle is passed from the driver to the computer and back again, or in the case of autonomous decision systems, how the vehicle would interact with other vehicles in any platoon or convoy in which the vehicle is travelling. More importantly, such controllers must await improvements in sensor technology and a vehicle's ability to detect its environment. Aggressive lateral manoeuvres at high speed should never be undertaken lightly.

\section{Acknowledgements}

This research is supported by the EU Sixth Framework Programme specific targeted research project Complex Embedded Automotive Control Systems (CEMACS), under contract 004175. The authors are grateful to Dr. Jens Kalkkuhl and Avshalom Suissa of DaimlerChrysler AG for useful discussions.

\section{References}

G. Burgio \& P. Zegelaar, "Integrated vehicle control using steering and brakes", Int. J. Control, 79, pp. 534-541, May 2006.

C. Canudas de Wit \& P. Tsiotras, "Dynamic tire friction models for vehicle traction control", in Proceedings of the IEEE Conference on Decision and Control, Vol. 4, 1999, pp. 3746-3751.

S. Germann, M. Würtenberger \& A. Daiß, "Monitoring of the friction coefficient between tyre and road surface", in Proceedings of the IEEE Conference on Control Applications, Part 1, Vol. 1, 1994, pp. 613618.

Y. Hattori, E. Ono \& S. Hosoe, "Optimum vehicle trajectory control for obstacle avoidance problem", IEEE-ASME T. Mech., 11 pp. 507-512, October 2006.

ISO-3888-2:2002, "Passenger cars - test track for a severe lane-change manoeuvre - part 2: Obstacle avoidance".

D.J. Leith, \& W.E. Leithead, "Gain-scheduled and nonlinear systems: dynamic analysis by velocity-based linearization families", Int. J. Control, 70, pp. 289-317, May 1998.

D.J. Leith, A. Tsourdos, B. White \& W. Leithead, "Application of velocity-based gain-scheduling to lateral auto-pilot design for an agile missile", Control Eng. Pract., 9, pp. 1079-1093, 2001.

W.F. Milliken \& D.L. Milliken, Race car vehicle dynamics, SAE International., 1995.

H.B. Pacejka \& E. Bakker, "The magic formula tyre model", Vehicle Syst. Dyn., 21 suppl., pp. 1-18, 1993. 
R. Rajamani, H.-S. Tan, B.K. Law \& W.-B. Zhang, "Demonstration of integrated longitudinal and lateral control for the operation of automated vehicles in platoons", IEEE T. Contr. Syst. T., 8, pp. 695-708, July 2000.

Z. Shiller \& S. Sundar, "Emergency lane-change manoeuvres of autonomous vehicles", J. Dyn. Syst.-T. ASME, 120, pp. 37-44, March 1998.

D. Swaroop \& S.M. Yoon, "Integrated lateral and longitudinal vehicle control for an emergency lane change manoeuvre design", Int J. Vehicle Des., 21, pp. 161-174, 1999.

A. Vahidi \& A. Eskandarian, "Research advances in intelligent collision avoidance and adaptive cruise control", IEEE T. Intell. Transp., 4, pp. 143-153, September 2004. 


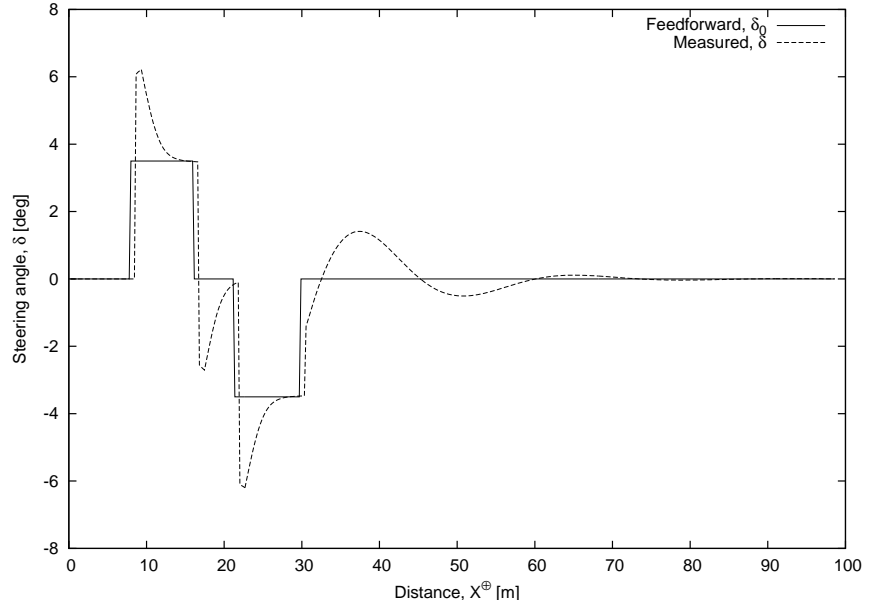

(a) Steering angle

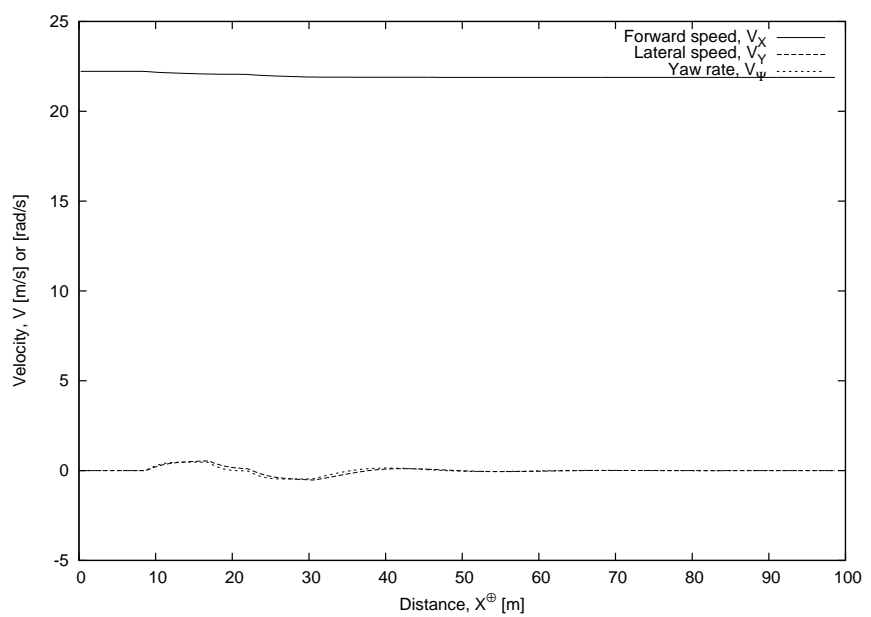

(c) Velocity

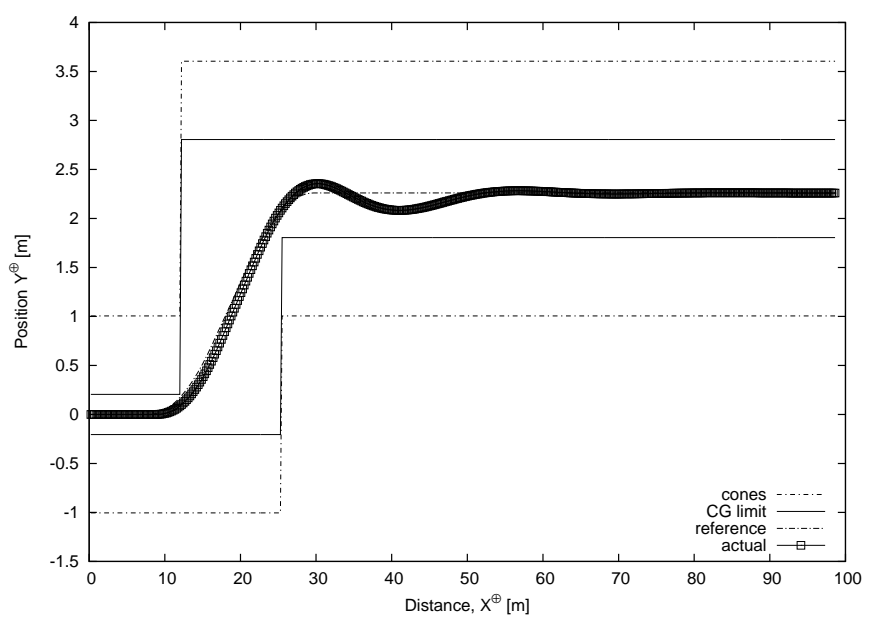

(e) Vehicle trajectory

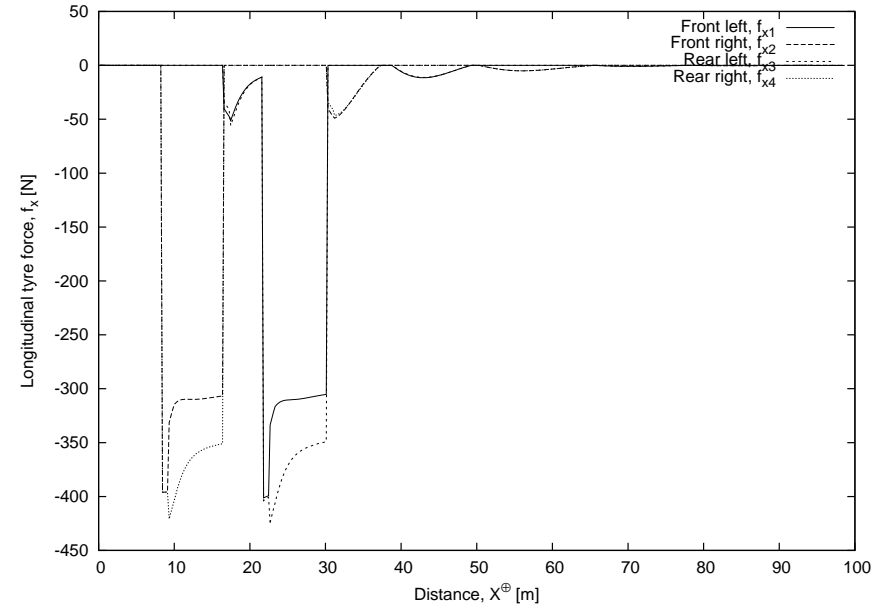

(b) Brake forces

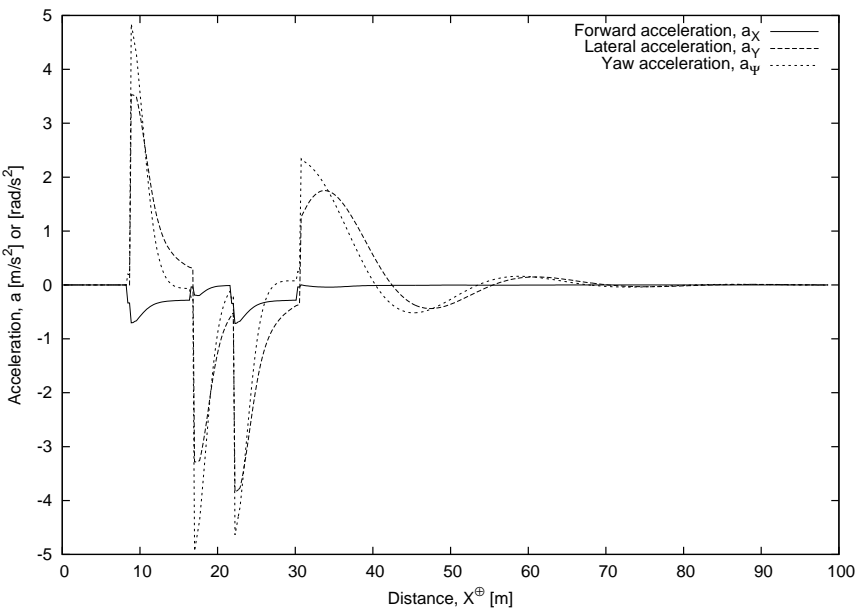

(d) Acceleration

Figure 9. Avoidance manoeuvre simulation at $80 \mathrm{kph}$ using integrated control 